\title{
PAMAM Dendrimer as Potential Delivery System for Combined Chemotherapeutic and MicroRNA-21 Gene Therapy
}

\author{
Xuan Zhou, Yu Ren, Xubo Yuan, Peiyu Pu and Chunsheng Kang \\ Department of Neurosurgery, Laboratory of Neuro-Oncology, Tianjin Medical University \\ Tianjin research center of basic medical science, Tianjin medical university \\ First Department of Head and Neck Cancer \\ Tianjin Medical University Cancer Institute $\mathcal{E}$ Hospital \\ School of Materials Science E Engineering, Tianjin University
}

China

\section{Introduction}

Chemotherapeutic drugs are fundamental in cancer management and are responsible for most cases of adjuvant treatment in patients after surgical procedures. However, the median overall survival does not increase in patients treated by concurrent chemo-radiotherapy. Consequently, further studies that could enhance the therapeutic effect should be encouraged [1].

Although the biological functions of microRNAs (miRNA) are not completely revealed, there is growing evidence that miRNA pathways are a new mechanism of gene regulation in both normal and diseased conditions [2]. Recent evidence has shown that miRNA mutations or aberrant expression patterns correlate with various diseases, such as cancer, viral infections, cardiovascular and indicates that miRNAs can function as tumor suppressors and oncogenes and oretically become a target to enhance the chemotherapeutic effect in cancer therapy.

However, although much work has been accomplished, the development of an efficient delivery system still remain a major challenge for the wide application of miRNA. In the following sections -- after a brief introduction of the miRNA strategies -- the potential and contributions of dendrimers in the development of effective non-viral delivery systems for combined the microRNA therapy with drug delivery will be discussed.

\section{MicroRNA strategy}

MicroRNAs (miRNAs) are a class of naturally occurring small non-coding RNAs, approximately 22 nucleotides in length, that target protein-coding mRNAs at the posttranscriptional level [4]. Generally, mature miRNAs are integrated into a protein-RNA complex called a microRNA RNPs (similar to the RISCs (RNA-induced silencing complexes) for siRNA). miRNAs bind through partial sequence homology to the 3_-untranslated regions ( $3^{\prime}$-UTRs) of target genes. Because of this unique feature, a single miRNA has 
multiple targets. It is thought that more than $30 \%$ of human genes are posttranscriptionally regulated by miRNAs [5]. miRNAs have diverse functions in biological processes, including the regulation of cellular proliferation, differentiation, and cell death. As dysregulation of these biological processes frequently occur in human cancer, miRNAs may, therefore, play a critical role in the process of tumorigenesis.

This regulatory mechanism was first shown in the developmental processes in worms, flies, and plants [6]. Subsequently, miRNAs have been shown to have important roles in many physiological processes of mammalian systems by influencing cell apoptosis, development, and metabolism through regulation of critical signaling molecules including cytokines, growth factors, transcription factors, and pro-apoptotic and anti-apoptotic proteins. Increasing number of miRNAs have been identified in the human genome and they are collectively called the miRNome [7]. Accumulating evidence shows the potential involvement of altered regulation of miRNAs in initiation and progression in a wide range of human cancers. Altered expression profiles of miRNAs are associated with genetic and epigenetic alterations including deletion, amplification, point mutation, and aberrant DNA methylation.

\section{1 miR-21 general aspects}

MiR-21 has been identified as the best hit in a number of miRNA profiling studies designed for the detection of miRNAs dysregulated in human cancer [8]. MiR-21 could suppress several tumor suppressor proteins translation, including PTEN, PDCD4, TMP1 and p53, to mediated cancer cell malignant phenotype alternation [9-10]. MiR-21 was emerging as key regulators of multiple pathways involved in tumorgenesis and may become the next targeted therapies in human cancers [11]. Previously we identified miR21's aberrant expression in glioblastoma(GBM), and we focused on in what way miR-21 regulated GBM development and progression. Thus, in the current chapter, we manage to elucidate the view point that miR-21 was an effective molecule with great potential of human cancer gene therapy; of course, we will take miR-21's biological role to GBM as an example.

Recent reports suggested that miR-21 functions as an oncogene in human cancers. Ciafre` et alprofiled the expression of 245 miRNAs in 10 glioblastoma (GBM) cell lines and nine freshly resected GBM samples and observed that miR-21 was overexpressed in human brain tumors [12]. It was shown that when miR-21 was suppressed, cell growth inhibition and caspase-dependent apoptosis were observed in A172, U87, LN229, and LN308 cells. It has been shown that miR-21 modulates breast cancer cell anchorage-independent growth through suppressing TMP1 expression. In human colorectal, breast cancer, and renal cell carcinoma, miR-21 contributes to invasion and metastasis cell by inhibiting Pdcd4 mRNA at the post-transcription level. A recent study showed that miR-21 targets PTEN gene through a binding site on the 3'-UTR in hepatocellular carcinoma [13]. PTEN has been shown to be a critical tumor suppressor gene that is commonly inactivated in GBM by deletion, mutation, or attenuated expression. Thus, increased expression of miR-21 may contribute to the attenuated expression of PTEN in GBM.

\section{2 miR-21 was up regulated in GBM cell lines and tissue samples}

Microarray assay was used to screen the miRNA expression status in GBM cell lines. Data showed miR-21 exhibited a 7.0-fold increase relative to normal brain tissue [14]. In addition, 
in-situ hybridization (ISH) of surgery resected glioma samples proved that miR-21 displayed varying degrees of intensity in glioma with different grades and the positive rate increased with the ascending order of the glioma WHO grade. In hence, it was important to note that miR-21 ISH was conducted at both the tissue level and the cellular level to indicate that miR-21 disregulation could be a marker to predict the outcome of glioma patients.

To identify miR-21 that was abnormal upregulated in high-grade gliomas, we used ISH to test miR-21 insitu expression in human non-neoplastic brain tissues, I II grade gliomas, grade III gliomas (anaplastic gliomas, AAs) and GBMs.

Our group showed that miR-21 was over expressed in 57 of 60 glioma samples and miR-21 was detected in the cytoplasm of the neoplastic cells of all the positive cases. MiR-21 displayed varying degrees of intensity in glioma with different grades and the positive rate increased with the ascending order of the WHO grade. There were 27 of $30(90 \%)$ in WHO I and II gliomas, 15 of $15(100 \%)$ in AAs and GBMs, whereas miR-21 was rarely detected in control brain tissues. The first indication of miR-21's aberrant expression came from the miRNA profiling of human glioblastoma. Compared to normal brain tissue, miR-21 relative expression was seven to eleven folds in low-grade astrocytomas, AAs and GBMs. Besides providing the consistent data to the previous study, it is important to note that miR-21 ISH was conducted at both the tissue level and the cellular level to indicate that miR-21 disregulation could be a marker to predict the outcome of glioma patients.

\section{3 miR-21 regulated GBM cell growth in vitro and in vivo}

To evaluate the significance of miR-21 overexpression in glioma cells, we used a loss-offunction antisense approach. An As-miR-21 oligonucleotide (ODN) was used to knock down miR-21 expression in U251 and LN229 cells. RT-realtime PCR results determined that the relative expression level of miR-21 in As-miR-21 ODN-treated U251 cell was 6.25\% $(\mathrm{P}<0.01)$ and $12.5 \%$ for LN229 cells $(\mathrm{P}<0.01)$ compared with their control cells, respectively. In addition, LNA-based in situ hybridization showed that transfection of a scrambled ODN had no effect on miR-21 expression. In contrast, the cy3 red fluorescence signal in As-miR-21-transfected U251 cells was lower (Figure 1B). These data suggested that As-miR-21 can specifically inhibit the endogenous miR-21 expression in U251 and LN229 cells.

The GBM cell growth inihibitory effect (MTT assay) of decreased miR-21 reached maximum three days post transfection. G1 phase blockage was observed to indicate cell cycle distribution changed significantly after miR-21 inhibitory. Additionally, the Annexin V positive early phase apoptotic cells were significantly increased in cells transfected with ASmiR-21 as compared to that in parental cells and cells treated with scrambled ODN.

The in vitro experiments suggest that miR-21 is a potential target for therapy in GBM. To further confirm this, we performed a proof-of-principle experiment using a U251 glioma xenograft model and a lipofectamine-mediated gene therapy approach. The xenograft tumors volume suppression indicated that miR-21 contributed a lot to U251 GBM cell proliferation in vivo. Pathological examination found micro-vessel density indicated that was and evaluated miR-21 expression by in situ hybridization and cellular apoptosis by TUNEL assay.

Despite the apparently predominance of microRNA in cancer therapy, several problems have to be overcome for successful clinical application. They show a poor stability towards nuclease activity, low intracellular penetration and low bioavailability. Although chemical modifications were brought to the basic microRNA, their sensitivity to degradation and poor intracellular penetration is still hampering their widespread clinical applications. In fact, the major bottleneck in the development of miRNA strategy is the delivery of these 
macromolecules to the target cells, tissues or organs. Therefore the development of more efficient delivery systems is regarded as one of the most promising strategies to solve these pharmaceutical hurdles. Specifically, delivery vectors must be designed to effectively complex with nucleic acid molecules and aid in overcoming intracellular barriers such as endosomal escape and cytoplasmic vector dissociation. For that reason, improvements on effective delivery have progressed rapidly. Among the different approaches under study, dendrimers are attracting a great interest for their well defined structure and great versatility in their chemistry that offer a unique platform for the rational design of efficient antisense delivery systems.

A

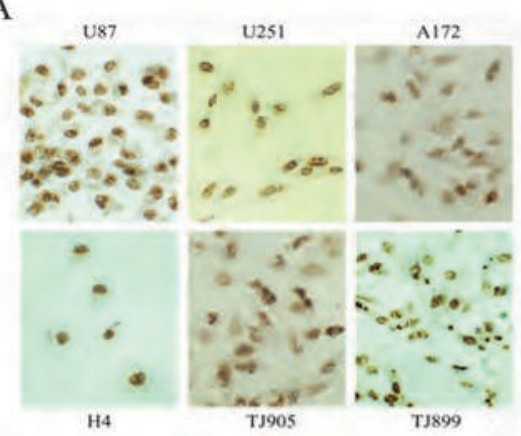

B

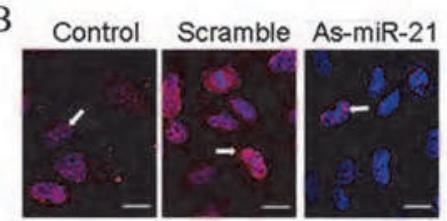

$\mathrm{E}$

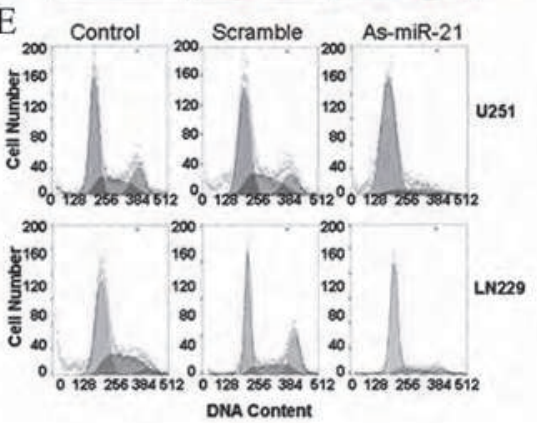

$\mathrm{C}$
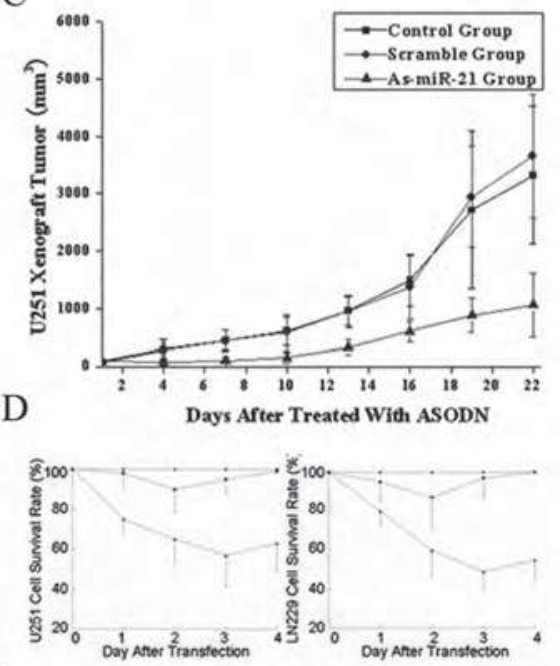

$\mathrm{F}$
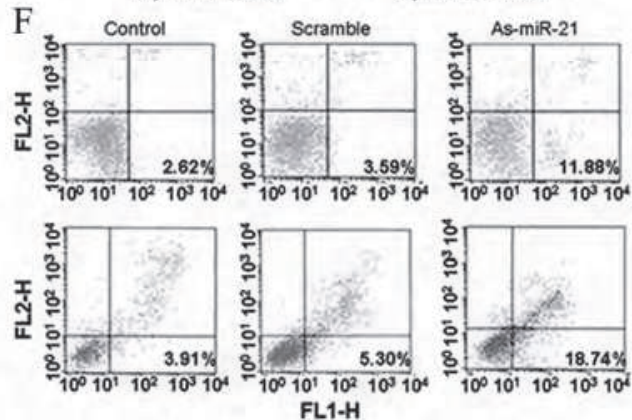

Fig. 1. The effect of miR-21 knockdown on U251 and LN229 GBM cell proliferation. (A) miR-21 was overexpressed in six glioma cells. (B) In situ examination of miR-21 expression in U251 cells. Arrows highlight miR-21 in situ expression in U251 cells. Bar $1 / 420 \mathrm{~mm}$. (C、D) MTT cell proliferation assay. miR-21 knockdown in U251 and LN229 GBM inhibits cell proliferation in vitro and in vivo. (E) Cell-cycle profiles after PI staining. miR-21 knockdown induced G1 arrest in both U251 and LN229 GBM cells. (F). As-miR-21 and scramble ODN-transfected U251 and LN229 GBM cells were analyzed using FCM to determine cell-cycle status. 


\section{Polyamidoamine (PAMAM) dendrimers}

Recently, a great deal of attention has been given to polyamidoamine (PAMAM) dendrimers; these are one of the most appropriate candidates for suitable carrier systems. PAMAM dendrimers represent an exciting new class of macromolecular architecture called dense star polymers. Unlike classical polymers, dendrimers have a high degree of molecular uniformity, a narrow molecular weight distribution, specific size and shape character [15]. The terminal amine groups of PAMAM dendrimers can be modified with different functionalities and can be linked with various biomolecules. These unique structural features of PAMAM dendrimers make them ideal nanoplatforms to conjugate biologically important substances.

\subsection{PAMAM dendrimer in gene transfection}

Gene therapy is a promising approach for the treatment of cancer because it enables the production of bioactive agents or the cessation of abnormal functions in the tumor cell. However, the success of gene therapy requires efficient and safe transfer systems because of the degradation of the delivered gene in the systemic circulation.

A variety of molecules including polymers, lipids, and peptides have been studied for their effectiveness as delivery vectors for DNA and RNA molecules. Successful delivery vectors must exhibit a combination of functional attributes. Polymeric carrier molecules should be cationic to complex with nucleic acids, possess a high buffering capacity, exhibit low cytotoxicity, and also contain chemically reactive groups that can be modified for the addition of targeting moieties or other groups.

As a non-viral gene delivery carrier, highly branched, dendritic polymers including poly(amidoamine) (PAMAM) have recently attracted interest as nucleic acid delivery vectors. Previous work has demonstrated that dendrimers can bind to DNA and RNA molecules and mediate modest cellular delivery of these nucleic acids. (PAMAM) dendrimers have attracted great interest due to their high efficacy in vitro gene delivery because of their branched structure. These dendritic polymers bear primary amine groups on their branched surface, which can bind DNA, compact it into polyplexes, and promote the cellular uptake of genes. Therefore, PAMAM dendrimers show high levels of transfection in a wide variety of cultured cells, especially in fractured form of G5 (commercially named SuperFect). Enhanced transfection efficiency has been reported by surface modification of PAMAM with L-arginine. Moreover, the primary amines located on the surface of PAMAM make it possible to conjugate suitable ligands, such as Transferrin, for efficient brain-targeting gene delivery.

Studies that focus on the cell entry mechanisms for several nonviral vectors, including PAMAM dendrimers [16]. The cationic surface charge imparted to the complex through high dendrimer-DNA charge ratios is required for subsequent interaction with the anionic glycoproteins and phospholipids that reside on the cell membrane surface. This interaction initiates the interior movement of the dendrimer-DNA complex into the cell cytosol, either by passive transport caused by membrane perturbations or by endocytosis. Complexes formed without an excess cationic surface charge do not mediate high gene transfection efficiency, which furnishes support for the importance of the initial electrostatic interaction between the complex and cell membrane. Studies following the incorporation of radiolabeled DNA and/or dendrimer components into cells established that the uptake in most cells was primarily via an active endocytosis mechanism. Cells preincubated with 
inhibitors of endocytosis (i.e. cytochalasin B and deoxyglucose) or cellular metabolism (i.e. sodium azide) reduced the uptake that corresponded to lower transgene expression, regardless of cell type.

These dendrimers as nanocarriers possess the following advantages: (1) neutral surface of the dendrimer for low cytotoxicity; (2) existence of cationic charges inside the dendrimer (not on the outer surface) resulting in highly organized compact nanoparticles, which can potentially protect nucleic acids from degradation. Noteworthly, surface modified QPAMAM-NHAc dendrimer demonstrated enhanced cellular uptake of siRNA when compared with the internally cationic QPAMAM-OH dendrimer (degree of quaternization $97 \%$ ).

George's study shows PEG-G5 and PEG-G6 dendrimers, with PEG conjugation molar ratio at $8 \%$ (PEG to surface amine per PAMAM), can facilitate dramatic intramuscular gene delivery in neonatal mice [17]. Park's group concluded that di-arginine conjugation to PAMAM dendrimers can improve polyplex stability, ntra-nuclear localization, and transfection efficiency but also induce charge density- and generation-dependent cytotoxicity. Therefore, a novel strategy for highly densed arginine conjugation maintaining low cytotoxicity will be needed for the development of efficient gene delivery carriers [18].

\subsection{PAMAM dendrimer as drug delivery system}

Polymeric drug delivery can improve bioavailability and efficacy of therapeutics with intrinsically poor water solubility and high toxicity. Dendrimers, a class of highly branched polymers, are effective drug delivery vehicles due to their monodispersity and nanoscopic size. With each increase in dendrimer generation, the diameter increases linearly while the number of surface groups increases exponentially. These high density surface groups can be conjugated to drug molecules, targeting moieties and imaging agents, rendering dendrimers a versatile drug delivery platform. In addition, surface groups on dendrimers can be modified to modulate cytotoxicity and permeation across biological barriers.

During their synthesis, PAMAM dendrimers can be produced that are either anionic or cationic in nature, with "full generations" (ie. G1, G2) having amine terminal groups and "half generations" (ie, G0.5, G1.5) possessing carboxylic acid terminal groups. The size and charge of PAMAM dendrimers impact their cytotoxicity and transepithelial transport, with cationic dendrimers showing higher toxicity in vitro. Due to their intrinsically low cytotoxicity and appreciable transepithelial permeation characteristics across Caco-2 monolayers and everted rat intestinal sac models, anionic dendrimers show distinct advantages as vehicles for oral drug delivery, with higher generation dendrimers showing the greatest potential because of their large number of modifiable surface groups.

PAMAM has well-defined internal cavities and an open architecture, guest molecules can become directly encapsulated into the macromolecule interior through hydrophobic interactions. Drug-polymer conjugates are potential candidates for the selective delivery of anticancer agents to tumor tissue. The main advantages of conjugating drugs to polymeric carriers include an increase in water solubility of low soluble or insoluble drugs, and therefore, enhancement of drug bioavailability, protection of drug from deactivation and preservation of its activity during circulation, a reduction in antigenic activity of the drug leading to a less pronounced immunological body response, and the ability to provide passive or active targeting of the drug specifically to the site of its action.

Surface-modified dendrimers were predicted to enhance pilocarpine bioavailability [19]. The anticancer drugs adriamycin and methotrexate were encapsulated into PAMAM 
dendrimers (i.e. G=3 and 4) which had been modified with PEG monomethyl ether chains (i.e. 550 and $2000 \mathrm{Da}$ respectively) attached to their surfaces. A similar construct involving PEG chains and PAMAM dendrimers was used to deliver the anticancer drug 5fluorouracil. Encapsulation of 5-fluorouracil into $G=4$ increase in the cytotoxicity and permeation of dendrimers.

Dendrimers have ideal properties which are useful in targeted drug-delivery system. One of the most effective cell-specific targeting agents delivered by dendrimers is folic acid PAMAM dendrimers modified with carboxymethyl PEG5000 surface chains revealed reasonable drug loading, a reduced release rate and reduced haemolytic toxicity compared with the non-PEGylated dendrimer. A third-generation dendritic unimolecular micelle with indomethacin entrapped as model drug gives slow and sustained in vitro release, as compared to cellulose membrane control [20]. Controlled release of the Flurbiprofen could be achieved by formation of complex with amine terminated generation 4 (G4) PAMAM Dendrimers [21]. The results found that PEG-dendrimers conjugated with encapsulated drug and sustained release of methotrexate as compare to unencapsulated drug.

\section{Multifunctional dendrimer nanodevices: In vitro and in vivo testing}

\subsection{Target gene therapy to rat $\mathbf{C 6}$ glioma cells rhrough folate receptor-PAMAM}

Despite the progress in the PAMAM mediated gene delivery, few studies have investigated the suitability of PAMAM dendrimers for ASODN delivery in vivo, especially for brain gliomas. The purpose of the present study is to evaluate whether in vivo gene delivery by folate-PAMAM (G5) conjugates can inhibit the development of gliomas. We selected the EGFR gene as an antisense target and the rat C6 intracranial glioma model for the in vivo study. Synthetic foliated (FA-)PAMAM was complexed with EGFR ASODN, and then the gene transfection efficacy, dynamic uptake, and biological effects of the FA-PAMAM delivery system on C6 rat glioma cells were investigated both in vitro and in vivo. Our results showed that the FA-PAMAM dendrimer conjugates transported EGFR-ASODNs into glioma cells in vitro, and yielded a favorable therapeutic effect in vivo on administration by local perfusion. Therefore, FA-PAMAM may represent a potential delivery system for short oligonucleotides in glioma-targeted therapy [22].

We chose G5 PAMAM as the gene vector in the present study because its many surface amine groups enable efficient complex formation with ASODNs through charge-based interactions. Western blot analysis demonstrated the binding of G5 PAMAM to ASODNs, with an optimum ASODN/PAMAM ratio of 16:1. TEM analysis revealed that the complexes were $>70 \mathrm{~nm}$ in size, and this small size likely enabled the efficient transfer of ASODNs to cells that we observed by flow cytometry. We used ASODNs directly labeled with fluorescent probes, such that flow cytometry directly reflected the uptake of the ASODN by the tumor. ASODN uptake mediated by PAMAM increased twofold in comparison with oligofectamine. The high uptake of ASODNs resulted in significant down-regulation of EGFR, suggesting that PAMAM mediated high efficiency transfection of C6 tumor cells with ASODNs. This high transfection efficiency can be attributed to not only the small size of the complexes, but also to the 'proton sponge' effect of PAMAM,31 in which the acidification of tertiary amino groups on PAMAM in the endosome increases the osmotic pressure within the endosome, leading to the release of ASODNs into cytoplasm.

However, while nonderivatized PAMAM achieves high efficiency transfection, its low targeting efficiency needs to be improved. One strategy to achieve this is the 
derivatization of PAMAM with ligands. Various ligands such as folic acid, transferring, and lactoferrin have been conjugated to PAMAM, thus enabling efficient gene targeting to tumors or brain. We chose folic acid as the functional ligand with which to modify PAMAM because of its low immunogenicity, unlimited availability, functional stability, defined conjugation chemistry, and a favorable nondestructive cellular internalization pathway.23 More importantly, the receptor for folic acid is a cell-proliferation protein that is overexpressed in many types of cancer cells.32-34 The expression levels of folate receptor in tumors have been reported to be 100 - 300 times higher than those observed in normal tissue.35 Although some ambiguity surrounds the expression level of the folate receptor in brain tumors,36 our results demonstrate that conjugation with folic acid enhanced the uptake of ASOND/PAMAM complexes by tumor cells and resulted in greater inhibition of EGFR expression in comparison with the native dendrimer. The in vivo study also demonstrates the superiority of FAPAMAM over either PAMAM or oligofectamine as a vector for mediating ASODN gene therapy. Dynamic contrast MRI scanning indicated significant suppression of tumor growth 2 weeks after C6 cell implantation (Fig. 2), which prolonged the survival time of rats in the FA-PAMAMmediated therapeutic groups.

In the first place, we evaluated the efficiency of folate-PAMAM dendrimers conjugates (FAPAMAM) for the in situ delivery of therapeutic antisense oligonucleotides (ASODN) that could inhibit the growth of C6 glioma cells. Folic acid was coupled to the surface amino groups of G5-PAMAM dendrimer (G5D) through a 1-[3-(dimethylamino)propyl]-3ethylcarbodiimide bond, and ASODNs corresponding to rat epidermal growth factor receptor (EGFR) were then complexed with FA-PAMAM. At an ASODN to PAMAM ratio of 16:1, agarose electrophoresis indicated that antisense oligonucleotides were completely complexed with PAMAM or FA-PAMAM. The ASODN transfection rates mediated by FAPAMAM and PAMAM were superior to oligofectamine, resulting in greater suppression of EGFR expression and glioma cell growth. Stereotactic injection of EGFR ASODN:FAPAMAM complexes into established rat C6 intracranial gliomas resulted in greater suppression of tumor growth and longer survival time of tumor-bearing rats compared with PAMAM and oligofectamine-mediated EGFR-ASODN therapy. The current study demonstrates the suitability of folate-PAMAM dendrimer conjugates for efficient EGFR ASODN delivery into glioma cells, wherein they release the ASODN from the FA-PAMAM to knock down EGFR expression in C6 glioma cells, both in vitro and in vivo. FA-PAMAM may thus represent a novel delivery system for short oligonucleotides in glioma-targeted therapy.

\subsection{Co-delivery of as-mir-21 and 5-fu by poly(amidoamine) dendrimer attenuates human glioma cell growth in vitro}

The efficacy of conventional chemotherapy is limited owing to the low therapeutic index of many anticancer drugs, as well as intrinsic or acquired drug resistance. To circumvent these difficulties, novel therapeutic strategies have been developed, and one attractive strategy is the combination of gene therapy with chemotherapy.

MicroRNAs have been demonstrated to be deregulated in different types of cancer. miR-21 is a key player in the majority of cancers. Down-regulation of miR-21 in glioblastoma cells leads to repression of cell growth, increased cellular apoptosis and cell-cycle arrest, which can theoretically enhance the chemotherapeutic effect in cancer therapy. 


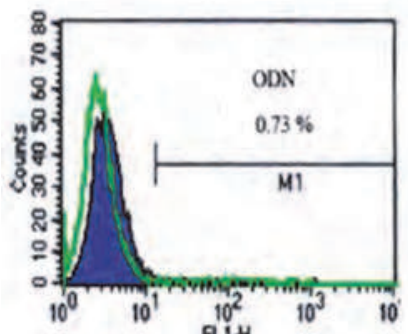

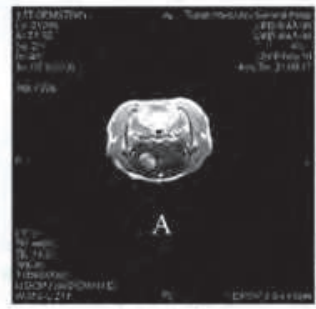

A: one week

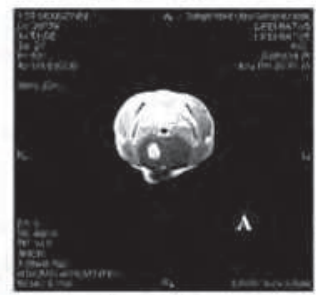

A: one week

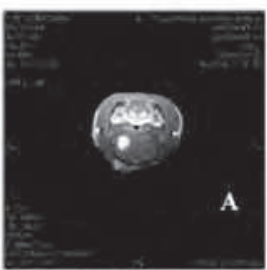

A: one week

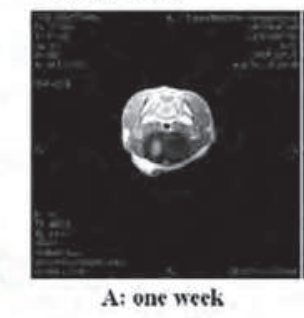

A: one week

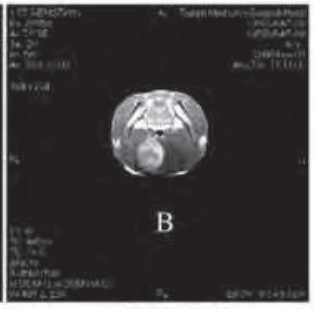

B: two weeks

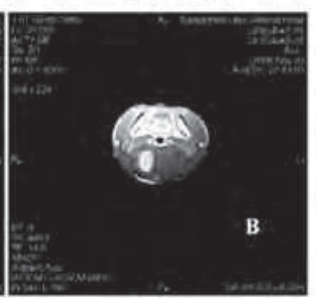

B: twe weeks
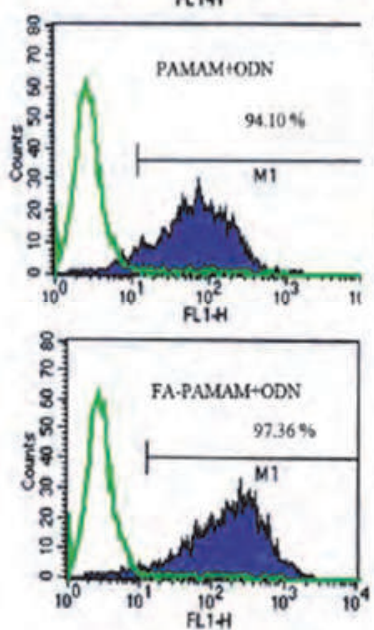

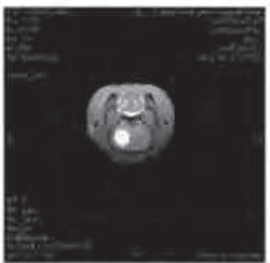

B: two weeks

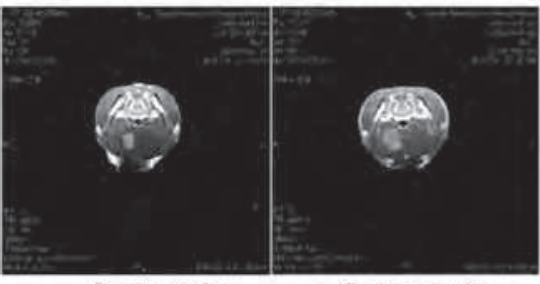

B: two weeks

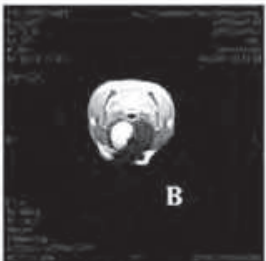

C: three weeks

C: three weeks

Fig. 2. Contrast-enhanced coronal MR images of representative animals in the ASODN, Oligofectamine/ASODN, PAMAM/ASODN, FA-PAMAM/ASODN group at 1, 2, and 3weeks after tumor xenograft. Four animals in PAMAM/ASODN and FAPAMAM/ASODN reached the 3-week time point, preventing a valid statistical comparison at that interval. The tumor in this animal is smaller in diameter and less contrast-enhanced than the one in the animal without gene therapy.

With positively charged primary amino groups on the surface, the PAMAM dendrimer can feasibly interact with biomolecules to form complexes through charge-based interactions, and protect them from rapid degradation by cellular endo- and exonucleases. Thus, the PAMAM dendrimer may be suitable for gene transfer or oligonucleotide delivery. Besides, because PAMAM has well-defined internal cavities and an open architecture, guest 
molecules can become directly encapsulated into the macromolecule interior through hydrophobic interactions. In this study, the poly(amidoamine) (PAMAM) dendrimer was employed as a carrier to co-deliver antisense-miR-21 oligonucleotide (as-miR-21) and 5fluorouracil (5-FU) to achieve delivery of as-miR-21 to human glioblastoma cells and enhance the cytotoxicity of 5-FU antisense therapy.

Taking advantage of hydrogen-bond interaction, we encapsulated 5-FU in the PAMAM nanoparticles simply by a membrane dialysis method. the encapsulation efficiency and loading efficiency of the drug were determined by UV spectroscopy to be 66.21 and $31.77 \%$, respectively. Through their charge-based interactions, 5-FU-PAMAM could conjugate with as-miR-21. The co-delivery of as-miR-21 not only significantly improved the cytotoxicity and chemosensitivity of 5-FU and dramatically increased the apoptotic percentage of the U251 cells but also brought down the migration ability of the tumor cells. The inhibitory effect toward brain tumors was evaluated by MTT assay, and measurements of cell apoptosis and invasion using the human brain glioma cell line U251. PAMAM could be simultaneously loaded with 5-FU and as-miR-21, forming a complex smaller than $100 \mathrm{~nm}$ in diameter. Both the chemotherapeutant and as-miR-21 could be efficiently introduced into tumor cells. The co-delivery of as-miR-21 significantly improved the cytotoxicity of 5-FU and dramatically increased the apoptosis of U251 cells, while the migration ability of the tumor cells was decreased. These results suggest that our co-delivery system may have important clinical applications in the treatment of miR21-overexpressing glioblastoma.

We report the anticancer potential of a combination of 5-FU treatment and antisense miR-21 technology using PAMAM dendrimers. PAMAM dendrimers, an available co-carrier of chemotherapeutant and as-miR-21, could effectively deliver 5-FU and as-miR-21 simultaneously, forming complexes smaller than $100 \mathrm{~nm}$ in diameter. The small size of the complexes facilitated their effective uptake by tumor cells, so the chemotherapeutant and asmiR-21 could be synchronously introduced to glioma cell for combined actions. The codelivery of as-miR-21 significantly improved the cytotoxicity of 5-FU and dramatically increased the level of apoptosis of U251 cells; it also decreased the migration abilities of the tumor cells. Our results provide invaluable information regarding the future application of drug-polymer complexes combined with gene therapy for cancer treatments. Taken together, our findings suggest that the combination of 5-FU treatment and as-miR-21 might be a potential clinical strategy for cancer chemotherapy [23].

\subsection{MicroRNA-21 inhibitor sensitizes human glioblastoma cells to taxol using PAMAM dendrimer}

Chemotherapeutic drugs are fundamental in cancer management and are responsible for most cases of adjuvant treatment in patients with GBMs after surgical procedures. Recently, much attention has been focused on the use taxol on glioma, both in experimental studies and in clinical trails [24]. However, the median overall survival did not increase in patients treated by concurrent chemoradiotherapy.

The successful of anti-cancer treatment are often limited by the development of drug resistance. Consequently, further studies that could enhance the therapeutic effect of taxol should be encouraged.Recent work has highlighted the involvement of non-coding RNAs, microRNAs(miRNAs) in cancer development, and their possible involvement in the evolution of drug resistance has been proposed. 

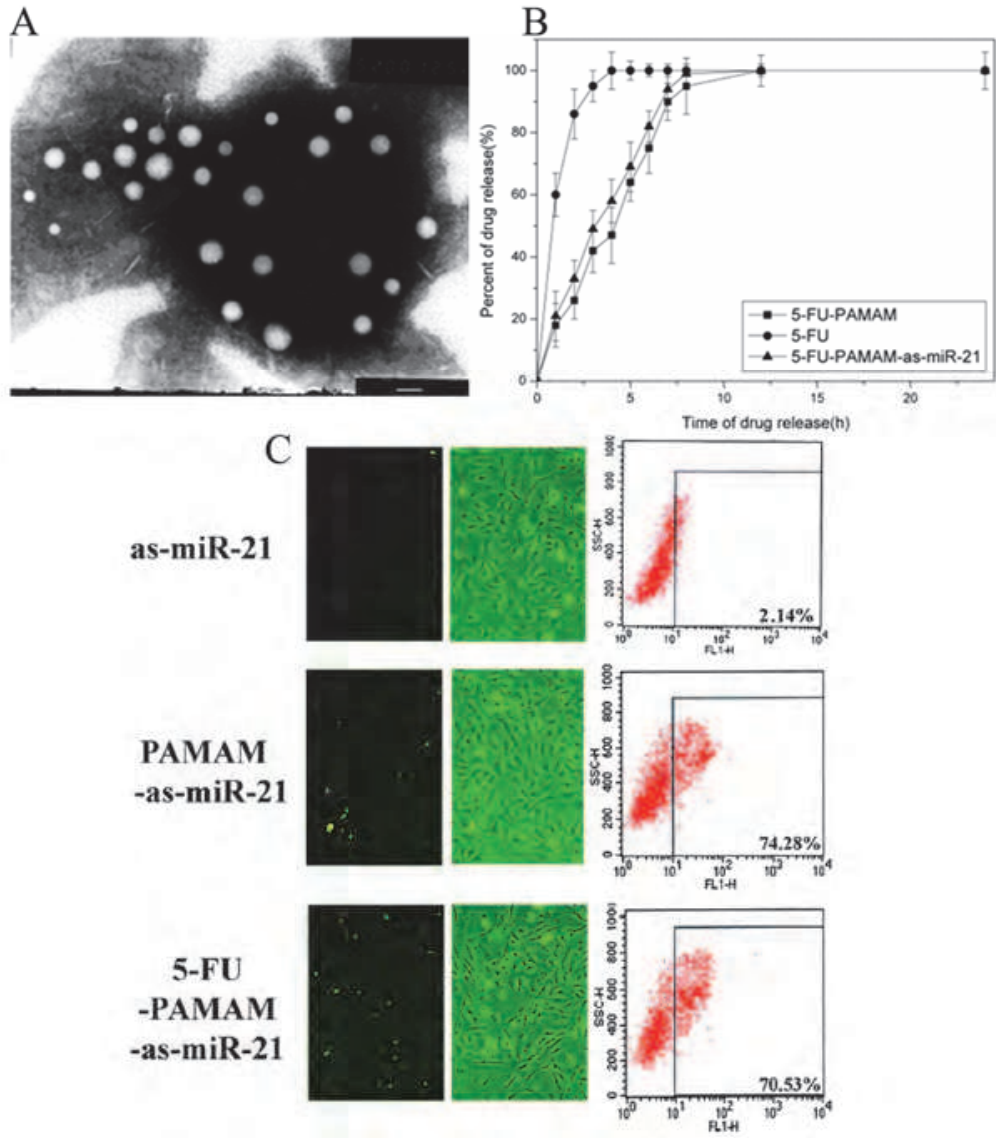

Fig. 3. Characterization of 5-FU/PAMAM/As-miR-21 complex. TEM image of 5-FUPAMAM-as-miR-21 complex, N/P = 16. Magnification $58000 \times$, scale bar $=100 \mathrm{~nm}(\mathrm{~A})$.

Release profiles of 5-FU from PAMAMand PAMAM-as-miR-21 complexes compared with free 5-FU (B). Cell uptake detected by flow cyctometry and fluorescent microscopy image of U251 cells after transfection with different complexes (C).

Substantial data indicate that the oncogene microRNA 21 (miR-21) is significantly elevated in glioblastoma multiforme (GBM) and regulates multiple genes associated with cancer cell proliferation, apoptosis, and invasiveness. Thus, miR-21 can theoretically become a target to enhance the chemotherapeutic effect in cancer therapy. So far, the effect of downregulating miR-21 to enhance the chemotherapeutic effect to taxol has not been studied in human GBM.

In this study, we combine taxol chemotherapy and miR-21 inhibitor treatment via polyamidoamine (PAMAM) dendrimers vector to evaluate the effects of combination therapy on suppression of glioma cells. The result indicated that the miR-21 inhibitor can decrease the proliferation of both U251 and LN229 cells and increase the cells' sensitivity to taxol treatment. The taxol concentration causing 50\% growth inhibition (IC50) of U251 cells is $400 \mathrm{nmol} / \mathrm{mL}$; whereas, in combination with the miR-21 inhibitor $(20 \mu \mathrm{mol} / \mathrm{L})$ the IC50 

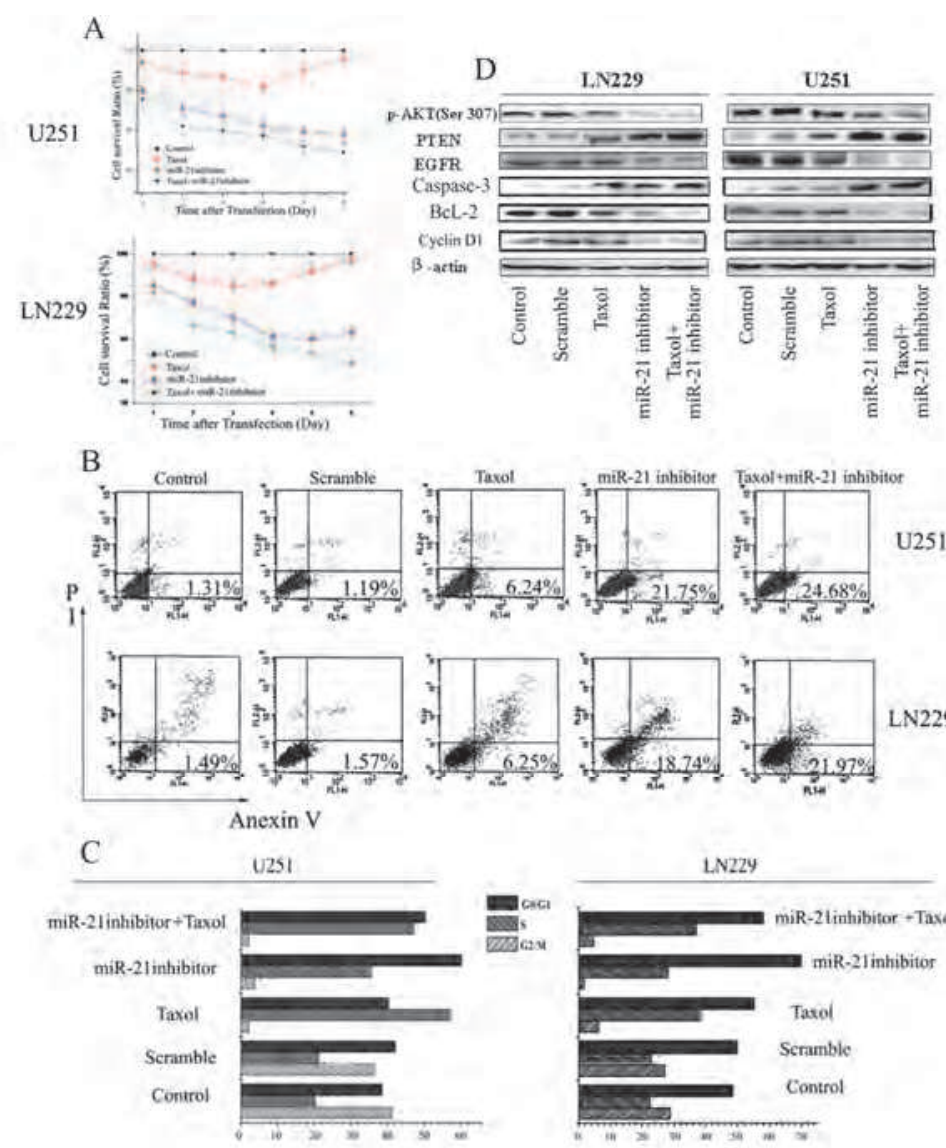

U251
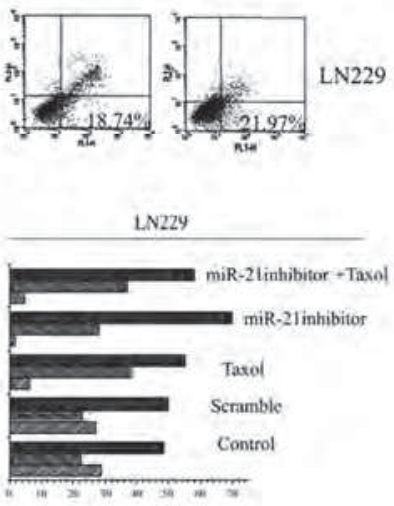

Fig. 4. Effect of the miR-21 inhibitor on the chemo-sensitivity of U251 and LN229 cells to taxol treatment. The growth of U251 and LN229 cells were inhibited by the miR-21 inhibitor, taxol only, and the indicated combinations. The cells were treated with the miR-21 inhibitor complexed to PAMAM for $6 \mathrm{~h}$ at $37^{\circ} \mathrm{C}$. The medium was then replaced with media containing various concentrations of taxol. After $72 \mathrm{~h}$ of incubation, an MTT assay was performed. Absorbance at $570 \mathrm{~nm}$ was normalized to the control (untreated cells) to determine cell viability. Each value represents the mean \pm SD from triplicate determinations. An aqueous solution of taxol (circles) and miR-21 inhibitor-loaded PAMAM (triangle) was incubated with human glioblastoma U251 and LN229 cells for six days. Druginduced decrease in cell numbers was measured using the MTT assay. The miR-21 inhibitor enhanced taxol induced apoptosis. Flow cytometry analyses of propidium iodide-stained cells were performed in triplicate (B). miR-21 inhibitor and taxol induce G1 and S phase arrest on cell cycle distribution. U251 and LN229 cells were treated with the miR-21 inhibitor and taxol alone or in combination, and cell cycle distributions were detected by Flow cytometry $48 \mathrm{~h}$ later (C). Evaluation of the expression of PTEN, EGFR, STAT3, and pSTAT3 in human glioblastoma LN229 and U251 cell lines. Western blot of protein extracts from cells treated with the miR-21 inhibitor or taxol, alone or combination (D). The expression of b-actin was examined to ensure uniform protein loading in all lanes. 
was $60 \mathrm{nmol} / \mathrm{mL}$. Taxol can also increase the efficacy of the miR-21 inhibitor. For example, combination treatment reduced cell viability to $20 \%$ compared with $86 \%$ viability for miR-21 inhibitor gene therapy alone. In LN229 cells, combination treatment with $20 \mu \mathrm{mol} / \mathrm{L}$ of the miR-21 inhibitor reduced the IC50 of taxol from 820 to $160 \mathrm{nmol} / \mathrm{L}$. It is worth noting that the miR-21 inhibitor additively interacted with taxol on U251cells and synergistically on LN229 cells.

Taxol treatment also increased the percentage of apoptotic cancer cells in miR-21 inhibitor transfected cells compared with control cells. Furthermore, treatment of the miR-21 inhibitor-transfected cells with the anti-cancer drugs taxol resulted in significantly reduced cell viability and invasiveness compared with control cells. These results indicated that the miR-21 plays an important role in the resistance of brain cancer cells to chemotherapeutic drugs. Therefore, miR-21 inhibitor gene therapy combined with taxol chemotherapy might represent a promising novel therapeutic approach for the treatment of glioblastoma.

Thus, the miR-21 inhibitor might interrupt the activity of EGFR pathways, independently of PTEN status. Meanwhile, the expression of STAT3 and p-STAT3 decreased to relatively low levels after miR-21 inhibitor and taxol treatment. The data strongly suggested that a regulatory loop between miR-21 and STAT3 might provide an insight into the mechanism of modulating EGFR/STAT3 signaling [25].

\section{Conclusion}

MiR-21 was one of the most frequently overexpressed miRNA in human glioblastoma (GBM) cell lines which can serve as a therapeutic target for glioblastoma. We validated that downregulation of miR-21 inhibited the growth of GBM cell lines and induced apoptosis. These effects were only partially dependent on PTEN, highlighting the existence of multiple, and possibly yet unknown, targets of miR-21. Inhibition of miR-21 also suppressed EGFR and Akt activity. These observations were confirmed in in vivo xenograft experiments that showed the potential clinical relevance of miR-21-targeting agents. Targeting miR-21 by antisense or small-molecule compounds may represent new targeted therapeutic strategies for human cancers, including gliomas.

PAMAM dendrimer has been reported to be good gene delivery candidate. Although the biological effects obtained from in vitro analysis of PAMAM and FA-PAMAM are approximate, our in vivo study implies that FAPAMAM is functionally effective for gene delivery into three-dimensional tissues. This may be due to folate-mediated targeting of ASODNs to folate receptor-expressing cells in solid tumors. Stereotactic administration, which enables FA-PAMAM-ASODNs to be injected directly into a tumor, may also produce better results than intravenous injection. Site-specific delivery remains the best choice to overcome gene delivery side effects and to increase its efficacy.

Next, we exhibit the anticancer potential of a combination of 5-FU treatment and antisense miR-21 technology using PAMAM dendrimers. PAMAM dendrimers, an available cocarrier of chemotherapeutant and as-miR-21, could effectively deliver 5-FU and as-miR-21 simultaneously, forming complexes smaller than $100 \mathrm{~nm}$ in diameter. The small size of the complexes facilitated their effective uptake by tumor cells, so the chemotherapeutant and asmiR-21 could be synchronously introduced to glioma cell for combined actions. The codelivery of as-miR-21 significantly improved the cytotoxicity of 5-FU and dramatically increased the level of apoptosis of U251 cells; it also decreased the migration abilities of the tumor cells. Our results provide invaluable information regarding the future application of 
drug-polymer complexes combined with gene therapy for cancer treatments. Taken together, our findings suggest that the combination of 5-FU treatment and as-miR-21 might be a potential clinical strategy for cancer chemotherapy.

Furthermore, the miR-21 inhibitor could enhance the chemo-sensitivity of human glioblastoma cells to taxol via PAMAM dendrimer. A combination of miR-21 inhibitor and taxol could be an effective therapeutic strategy for controlling. The above data suggested that in both the PTEN mutant U251 cell line and the PTEN wild-type LN229 cells, miR-21 blockage could increase the chemosensitivity to taxol. It is worth noting that the miR-21 inhibitor additively interacted with taxol on U251cells and synergistically on LN229 cells. Thus, the miR-21 inhibitor might interrupt the activity of EGFR pathways, independently of PTEN status. The miR-21 inhibitor enhanced the chemo-sensitivity of human glioblastoma cells to taxol and combination of the miR-21 inhibitor and taxol could be an effective therapeutic strategy for suppressing the growth of GBM.

the growth of GBM by inhibiting STAT3 expression and phosphorylation.

\section{Acknowledgements}

This work was financially supported by the China National Natural Scientific Fund (51073118 and 30971136), the Tianjin Science and Technology Committee (09JCZDJC17600, 10JCYBJC12500), and a Program for New Century Excellent Talents in University (NCET-070615).

\section{References}

Minniti G, et al. (2009). Chemotherapy for glioblastoma: current treatment and future perspectives for cytotoxic and targeted agents. Anticancer Res, Vol.29, No.12, (2009 Dec) pp: 5171-84, ISSN: 0250-7005

Santos-Rebouças CB,et al (2010). MicroRNAs: macro challenges on understanding human biological functions and neurological diseases. Curr Mol Med. Vol.10, No.8, (2010 Nov) pp: 692-704, ISSN: 1566-5240

Kaneda Y. (2010). Update on non-viral delivery methods for cancer therapy: possibilities of a drug delivery system with anticancer activities beyond delivery as a new therapeutic tool. Expert Opin Drug Deliv. Vol.7, No.9, (2010 Sep), pp: 1079-93, ISSN: 1742-5247.

He L, Hannon GJ. (2004) MicroRNAs: small RNAs with a big role in gene regulation. Nat Rev Genet. Vol. 5, No. 7, (2004 Jul), pp: 522-31, ISSN: 1471-0056

Bartel DP. (2004). MicroRNAs: genomics, biogenesis, mechanism, and function. Cell. Vol. 116, No. 2, (2004 Jan), pp: 281-97, ISSN: 2155-1790

Akbergenov R, et al (2006). Molecular characterization of geminivirus-derived small RNAs in different plant species. Nucleic Acids Res, Vol.34, (2006 Jan) pp: 462-471, ISSN: 0305-1048

Valeri N, et al (2009). Epigenetics, miRNAs, and human cancer: a new chapter in human gene regulation. Mamm Genome, Vol. 20, No. 9-10, (2009 Sep-Oct), pp: 573-80 , ISSN: 0938-8990

Selcuklu SD, Donoghue MT, Spillane C (2009). miR-21 as a key regulator of oncogenic processes. Biochem Soc Trans. Vol. 37, No. 4, (2009 Aug), pp: 918-25, ISSN: 0300-5127 
Zhu S, et al (2007). MicroRNA-21 targets the tumor suppressor gene tropomyosin 1 (TPM1). J Biol Chem, Vol. 282, No.19, (2007 May), pp: 14328-14336, ISSN: 0021-9258

Asangani IA, et al (2008). MicroRNA-21 (miR-21) post-transcriptionally downregulates tumor suppressor Pdcd4 and stimulates invasion, intravasation and metastasis in colorectal cancer. Oncogene, Vol. 27, No. 15, (2008 Apr), pp: 2128-2136, ISSN: 09509232

Moore LM, Zhang W. Targeting miR-21 in glioma: a small RNA with big potential. Expert Opin Ther Targets. Vol. 14, No.11, (2010 Nov r), pp: 1247-57, ISSN: 1472-8222

Ciafre SA, et al. Extensive modulation of a set of microRNAs in primary glioblastoma. Biochem Biophys Res Commun Vol. 334 , No.4 , (2005 Sep), pp: 1351-1358, ISSN: 0006-291X

Meng F, et al (2007). MicroRNA-21 regulates expression of the PTEN tumor suppressor gene in human hepatocellular cancer. Gastroenterology, Vol. 133, No. 2, (2007 Aug), pp: 647-658, ISSN: 0016-5085

Malzkorn B, et al (2010). Identification and functional characterization of microRNAs involved in the malignant progression of gliomas. Brain Pathol. Vol.20, No.3, (2010 May), pp: 539-50, ISSN: 1015-6305

Baker JR Jr (2009). Dendrimer-based nanoparticles for cancer therapy. Hematology Am Soc Hematol Educ Program. pp: 708-19, ISSN: 1520-4391

Eichman JD, et al (2000). The use of PAMAM dendrimers in the efficient transfer of genetic material into cells. Pharm Sci Technolo Today, Vol. 3, No. 7, (2000 Jul), pp: 232-245, ISSN: 1461-5347

Qi R, et al (2009). PEG-conjugated PAMAM dendrimers mediate efficient intramuscular gene expression. AAPS J. Vol. 11 , No. 3, (2009 Sep) pp: 395-405, ISSN: 1550-7416

Kim TI, et al (2009). Comparison between arginine conjugated PAMAM dendrimers with structural diversity for gene delivery systems. J Control Release. Vol. 136, No. 2, (2009 Jun) pp: 132-9, ISSN: 0168-3659

Tolia,G.T et al (2008). The role of dendrimers in drug delivery, Pharmaceut. Tech., Vol. 32, No.11, (2008 Nov) pp:88-98, ISSN:

Patri, A.K., Majoros and. Baker, J.R., (2002). Dendritic polymer macromolecular carriers for drug delivery, Curr. Opin. Chem. Biol. Vol. 6, No. 4, (2002 Aug) pp: 466-71, ISSN: 1367-5931

Asthana ,A.,et al, 2005 . Poly (amidoamine) (pamam) dendritic nanostructures for controlled site specific delivery of acidic anti-inflammatory active ingredient, AAPS PharmSciTech. Vol. 6 , No. 3, (2005 Oct) pp: E536-42, ISSN: 1530-9932

Kang C, et al (2010). Evaluation of folate-PAMAM for the delivery of antisense oligonucleotides to rat C6 glioma cells in vitro and in vivo. J Biomed Mater Res A. (2010 May), Vol. 93 , No. 2, pp: 585-94, ISSN: 1549-3296

Ren Y, et al (2010). Co-delivery of as-miR-21 and 5-FU by poly(amidoamine) dendrimer attenuates human glioma cell growth in vitro. J Biomater Sci Polym Ed. Vol. 21 , No. 3, (2010 Mar) pp: 303-14., ISSN: 0920-5063

Karmakar S, et al (2007). Combination of all- trans retinoic acid and taxol regressed glioblastoma T98G xenografts in nude mice. Apoptosis, Vol.12, No.11, (2007 Nov) pp: 2077-87, ISSN: 1360-8185. 
Ren Y, et al (2010). MicroRNA-21 inhibitor sensitizes human glioblastoma cells U251 (PTENmutant) and LN229 (PTEN-wild type) to taxol. BMC Cancer. Vol. 10 , (2010 Jan) pp: 27, ISSN: 1471-2407 


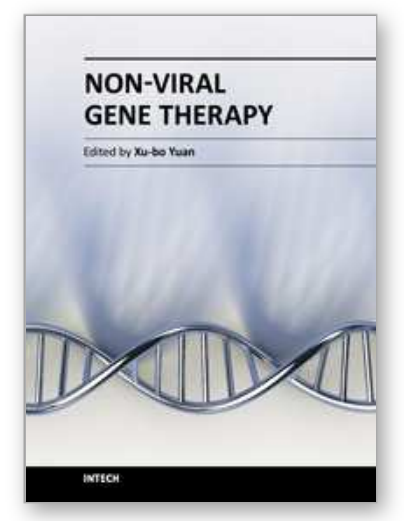

\author{
Non-Viral Gene Therapy \\ Edited by Prof. Xubo Yuan
}

ISBN 978-953-307-538-9

Hard cover, 696 pages

Publisher InTech

Published online 07, November, 2011

Published in print edition November, 2011

This book focuses on recent advancement of gene delivery systems research. With the multidisciplinary contribution in gene delivery, the book covers several aspects in the gene therapy development: various gene delivery systems, methods to enhance delivery, materials with modification and multifunction for the tumor or tissue targeting. This book will help molecular biologists gain a basic knowledge of gene delivery vehicles, while drug delivery scientist will better understand DNA, molecular biology, and DNA manipulation.

\title{
How to reference
}

In order to correctly reference this scholarly work, feel free to copy and paste the following:

Xuan Zhou, Yu Ren, Xubo Yuan, Peiyu Pu and Chunsheng Kang (2011). PAMAM Dendrimer as Potential Delivery System for Combined Chemotherapeutic and MicroRNA-21 Gene Therapy, Non-Viral Gene Therapy, Prof. Xubo Yuan (Ed.), ISBN: 978-953-307-538-9, InTech, Available from:

http://www.intechopen.com/books/non-viral-gene-therapy/pamam-dendrimer-as-potential-delivery-system-forcombined-chemotherapeutic-and-microrna-21-gene-ther

\section{INTECH}

open science | open minds

\section{InTech Europe}

University Campus STeP Ri

Slavka Krautzeka 83/A

51000 Rijeka, Croatia

Phone: +385 (51) 770447

Fax: +385 (51) 686166

www.intechopen.com

\section{InTech China}

Unit 405, Office Block, Hotel Equatorial Shanghai

No.65, Yan An Road (West), Shanghai, 200040, China

中国上海市延安西路65号上海国际贵都大饭店办公楼 405 单元

Phone: +86-21-62489820

Fax: +86-21-62489821 
(C) 2011 The Author(s). Licensee IntechOpen. This is an open access article distributed under the terms of the Creative Commons Attribution 3.0 License, which permits unrestricted use, distribution, and reproduction in any medium, provided the original work is properly cited. 